\title{
WORK AT THE SPEED OF THOUGHT
}

Take clinical efficiency to new levels through smart, personalized automation.

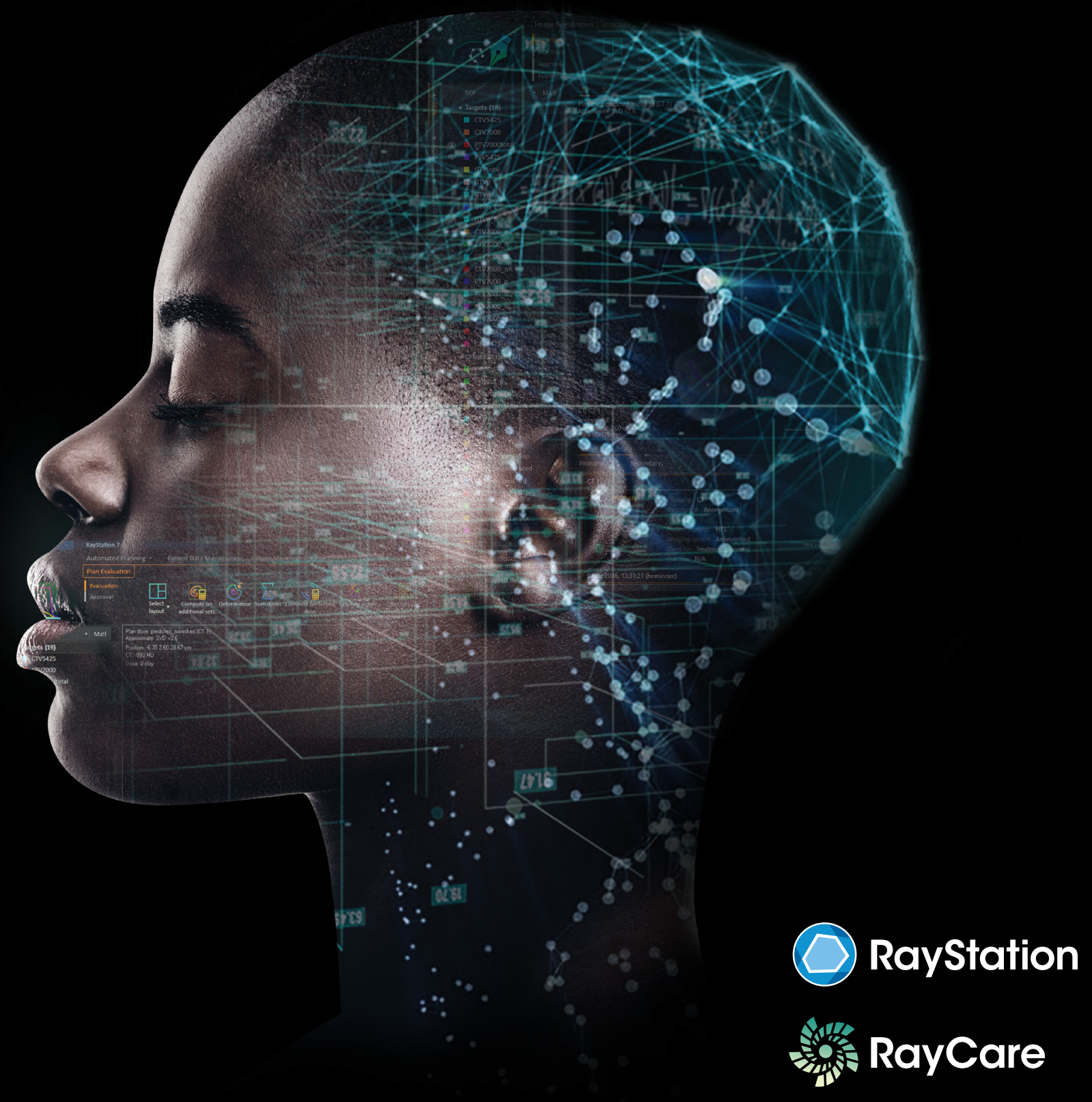

\section{ADVANCING CANCER TREATMENT}

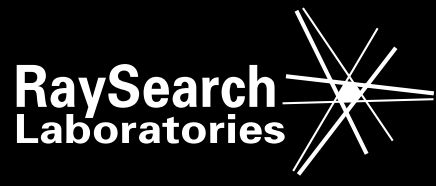




\title{
Feasibility of Improving the Accuracy of Dose Calculation Using Hybrid Computed Tomography Images: A Phantom Study
}

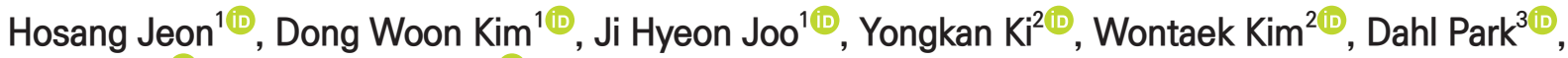 \\ Jiho $\mathrm{Nam}^{3(\mathbb{1})}$, Dong Hyeon $\mathrm{Kim}^{3 \text { (10 }}$
}

'Department of Radiation Oncology and Research Institute for Convergence of Biomedical Science and Technology, Pusan National University Yangsan Hospital, Yangsan, ${ }^{2}$ Department of Radiation Oncology, Pusan National University School of Medicine, Yangsan, ${ }^{3}$ Department of Radiation Oncology, Pusan National University Hospital, Busan, Korea

Received 30 December 2020 Revised 13 January 2021

Accepted 19 January 2021

Corresponding author

Yongkan Ki

(apex7171@hanmail.net)

Tel: 82-55-360-3450

Fax: 82-55-360-3449
Purpose: Kilovoltage computed tomography $(\mathrm{kV}-\mathrm{CT})$ is essential for radiation treatment planning. However, kV-CT images are significantly distorted by artifacts when a metallic prosthesis is present in the patient's body. Thus, the accuracies of target delineation and treatment dose calculation are inevitably lowered. We evaluated the accuracy of the calculated doses using an image restoration method with hybrid CT, which was introduced in our previous study.

Methods: A cylindrical phantom containing four metals, namely, silver, copper, tin, and tungsten, was scanned using $\mathrm{kV}-\mathrm{CT}$ and megavoltage CT to produce hybrid CT images. We created six verification plans for three head and neck patients on $\mathrm{kV}-\mathrm{CT}$ and hybrid $\mathrm{CT}$ images of the phantom and calculated their doses. The actual doses were measured with film patches during beam delivery using tomotherapy. We used the gamma evaluation method to compare dose distribution between $\mathrm{kV}-\mathrm{CT}$ and hybrid CT with three gamma criteria, namely, $3 \% / 3 \mathrm{~mm}, 2 \% / 2 \mathrm{~mm}$, and 1\%/1 $\mathrm{mm}$.

Results: The gamma pass rates decreased as the gamma criteria were strengthened, and the pass rate of hybrid CT was higher than that of $\mathrm{kV}-\mathrm{CT}$ in all cases. When the $1 \% / 1 \mathrm{~mm}$ criterion was used, the difference in gamma pass rates between them was up to $13 \% \mathrm{p}$.

Conclusions: According to our findings, we expect that the use of hybrid CT can be a suitable approach to avoid the effect of severe metal artifacts on the accuracy of dose calculation and contouring.

Keywords: Metal artifact, Hybrid computed tomography, Radiation treatment planning, Dose calculation

\section{Introduction}

In modern radiotherapy, kilovoltage computed tomography $(\mathrm{kV}-\mathrm{CT})$ is an essential imaging system for radiation treatment planning (RTP). kV-CT images are used for calculating therapeutic radiation doses absorbed by the patient's body and for delineating the target volume and the volume in the surrounding normal organs. However, kV-CT images are significantly distorted by image artifacts caused by the presence of metal prostheses, such as dental fillings or artificial knee joints in a patient's body. Thus, the accuracies of target delineation and treatment dose calculation are in- 
evitably lowered. Many studies on metal artifact reduction (MAR) in kV-CT images have been conducted since Glover and Pelc [1] first reported this issue in 1981. Most studies on MAR suggested similar procedures: (1) identification of metal inserts in raw projection data or reconstructed CT images; and (2) replacement of the identified data with the neighboring data through various interpolation techniques [2-8]. Recently, alternative trials based on iterative methods have been suggested [9-11]. Although the effects of these MAR studies were significant, the original pixel values of CT images near metal prostheses could not be restored because incident X-ray photons were fully absorbed or scattered during penetration. This means that the treatment dose values cannot be calculated accurately. However, megavoltage CT (MV-CT) [12,13] uses higher X-ray energy beams that can penetrate high-density materials and produces CT images with rare metal artifacts and accurate pixel values of metal prostheses. MV-CT images present higher noise and lower image contrast due to the high transmission and scat- tering properties of megavoltage X-ray energies, which may preclude precise image reading or identification of regions of interest.

In 2015, we proposed a novel method to generate hybrid CT images utilizing the advantages of kV-CT and MV-CT and demonstrated its benefits in terms of image quality using a cylindrical phantom in three clinical cases [14]. We showed that, like MV-CT, hybrid CT also successfully removed artifacts due to metal prostheses while maintaining high image quality compared to that of kV-CT. As a followup study, this study evaluates the accuracy of the calculated treatment dose when the calculation was implemented using hybrid CT and compares it with that of the original $\mathrm{kV}$-CT. MV-CT was excluded from this comparative study owing to its inherently low image quality. We expect that the feasibility of hybrid CT as an imaging technique for accurate radiotherapeutic dose calculation would be considered.
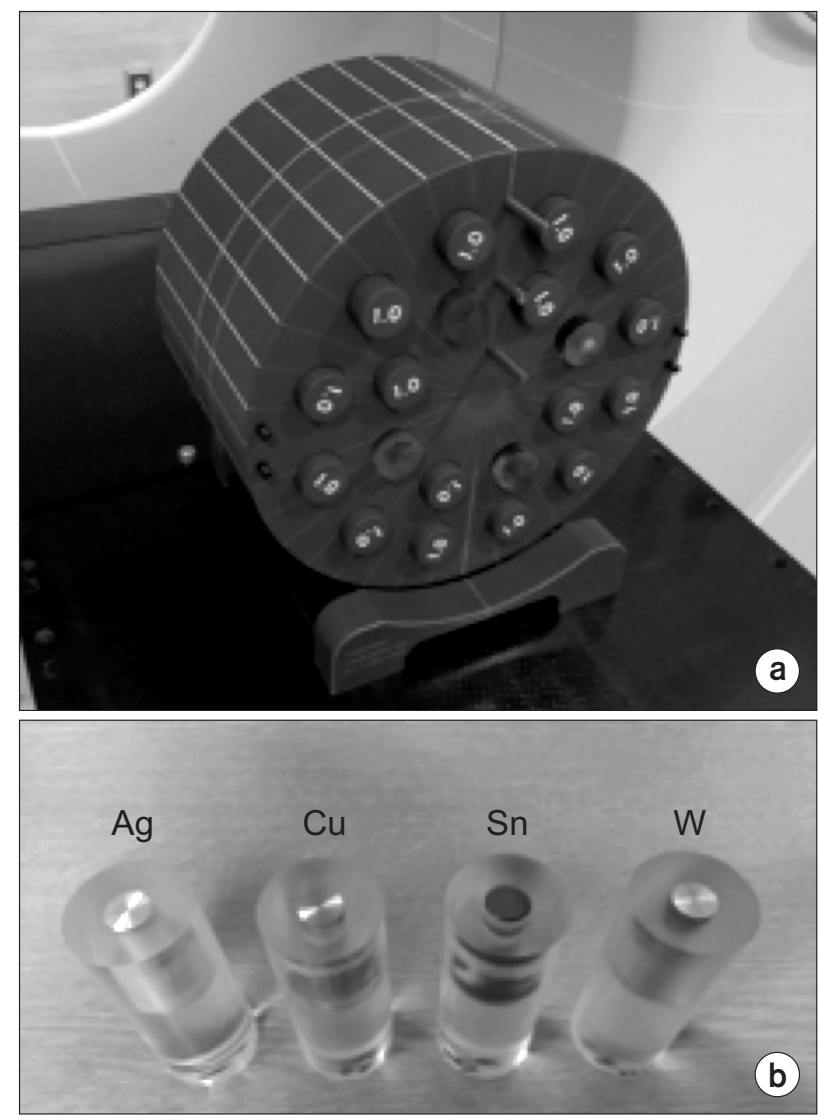

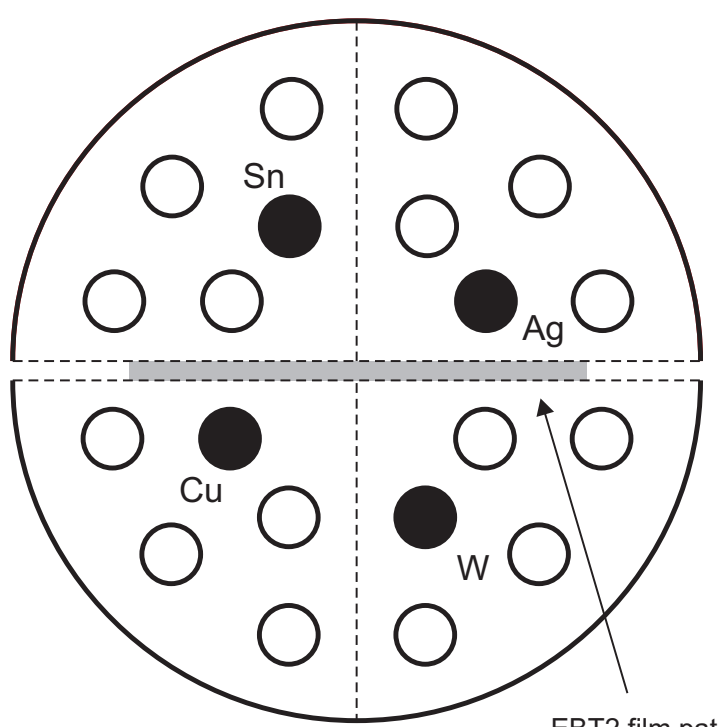

EBT2 film patch $\left(200 \times 30 \times 0.28 \mathrm{~mm}^{3}\right)$

(C)

Fig. 1. Experimental setup of (a) the cylindrical phantom containing (b) four metal inserts. (c) Transverse view of the phantom. Ag, silver; $\mathrm{Cu}$, Copper; $\mathrm{Sn}$, tin; W, tungsten. 


\section{Materials and Methods}

\section{Experimental setup}

A standard cheese phantom (TomoTherapy, Inc., Madison, WI, USA), which is a cylindrical solid water phantom with a diameter of $30 \mathrm{~cm}$, was used. The phantom contained four polymethyl methacrylate holders. They were embedded with silver (Ag, physical density=10.49 $\mathrm{g} / \mathrm{cm}^{3}$ ), copper $\left(\mathrm{Cu}, 8.94 \mathrm{~g} / \mathrm{cm}^{3}\right)$, tin $\left(\mathrm{Sn}, 7.37 \mathrm{~g} / \mathrm{cm}^{3}\right)$, and tungsten $\left(\mathrm{W}, 19.25 \mathrm{~g} / \mathrm{cm}^{3}\right)$ rods. These metals represent the main components in metal prostheses, such as dental amalgams and implants. The phantom was scanned using a 16-slice Brilliance Big Bore CT (Philips Healthcare, Andover, MA, USA) with a $3-\mathrm{mm}$ spacing, $250 \mathrm{~mA}$, and $120 \mathrm{kV}$ to obtain a $\mathrm{kV}$-CT image set. Besides, an MV-CT image set was scanned using a helical tomotherapy (HT) system (TomoTherapy,
Inc., Madison, WI, USA) with a 4-mm spacing and 3.5 MV. Fig. 1 shows the overall experimental setup. We inserted film patches (EBT2, Ashland, NJ, USA), in the horizontal direction, at the center of the phantom to measure dose distributions (Fig. 1c). The dose values measured on the film were read using a flatbed scanner (Expression 10000XL; Epson, Nagano, Japan).

\section{Hybrid CT}

A hybrid CT image set of the phantom was generated using the corresponding $\mathrm{kV}$-CT and MV-CT images according to the post-processing procedure reported in our previous study [14]. Metal-affected kV sinogram data were identified and replaced with the corresponding data of the MV sinogram after adequate calibration between the $\mathrm{kV}$ and $\mathrm{MV}$ sinograms. Fig. 2 shows the reconstructed images of $\mathrm{kV}-\mathrm{CT}$
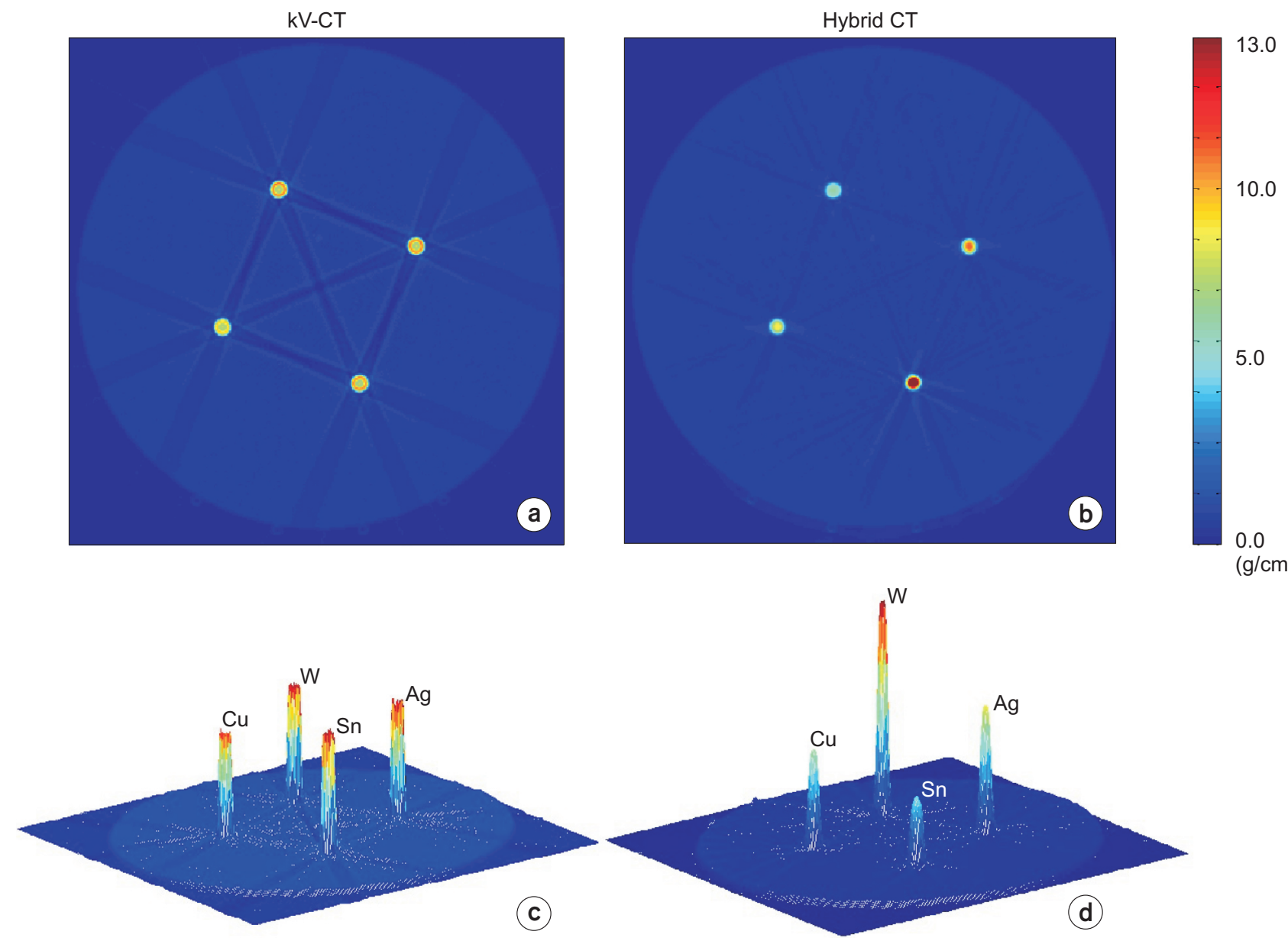

$\left(\mathrm{g} / \mathrm{cm}^{3}\right)$

Fig. 2. Density distributions of the phantom (a) scanned using kilovoltage computed tomography (kV-CT) and (b) generated using hybrid $\mathrm{CT}$, expressed in $\mathrm{g} / \mathrm{cm}^{3}$. (c, d) They are also plotted on three-dimensional axes. Ag, silver; Cu, Copper; Sn, tin; W, tungsten. 


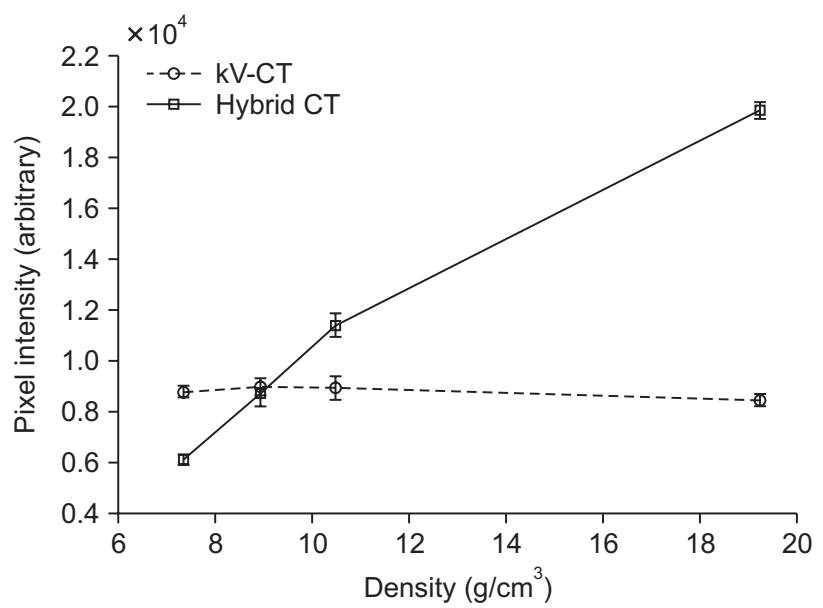

Fig. 3. Density-intensity curves in the reconstructed images of kilovoltage computed tomography $(\mathrm{kV}-\mathrm{CT})$ and hybrid CT.

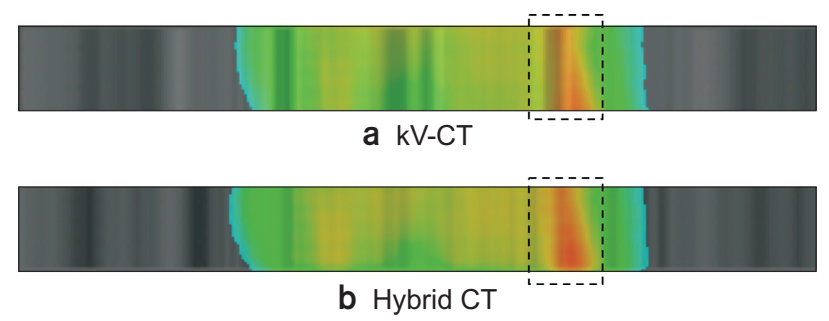

Fig. 4. Calculated dose distributions on the phantom obtained using (a) kilovoltage computed tomography (kV-CT) and (b) hybrid CT in the coronal view in patient 1 .

and hybrid CT. We observe that the pixel intensities of all metallic rods are saturated in $\mathrm{kV}-\mathrm{CT}$, and those in hybrid CT accurately reflect the real density of each metallic rod. Fig. 3 shows the plots of quantitative values.

\section{Dose calculation and analysis}

Three clinical HT plans for three head and neck patients (nasopharynx, submandible, and retromolar trigone) were selected to evaluate the hybrid CT performance. The prescribed dose for the three plans was $50 \mathrm{~Gy}$, with 25 fractionations, and optimization constraints were set to meet the clinical requirements of our physicians. We created six verification plans for the clinical plans on $\mathrm{kV}-\mathrm{CT}$ and hybrid CT of the phantom. A convolution/superposition algorithm using the collapsed-cone approach [15-17] was used to calculate the dose distribution with a calculation grid of $2 \mathrm{~mm}$. Fig. 4 shows the dose distributions calculated using kV-CT

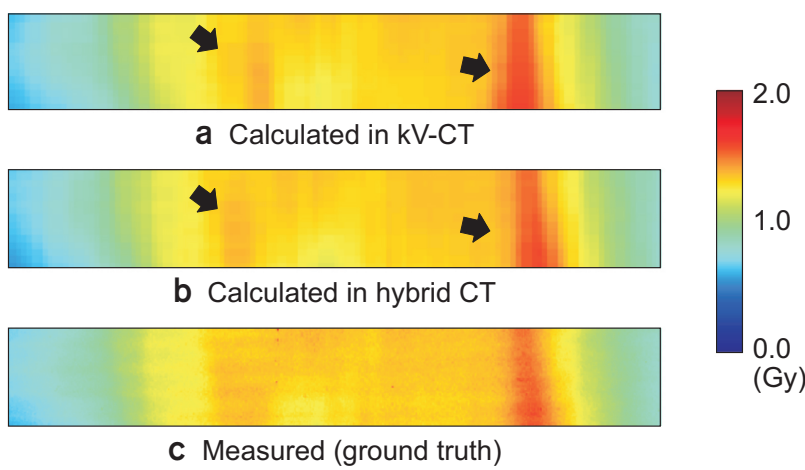

Fig. 5. Dose distributions calculated using (a) kilovoltage computed tomography (kV-CT) and (b) hybrid CT, and (c) measured using film in the coronal view in patient 1 . The size of the film is $200 \times 30 \mathrm{~mm}^{2}$.

and hybrid CT in patient 1 . The dark streak regions due to metal artifacts in $\mathrm{kV}-\mathrm{CT}$ and the resultant difference in the calculated dose distributions are well observed (see dotted boxes in Fig. 4).

The dose distributions calculated using $\mathrm{kV}-\mathrm{CT}$ and hybrid CT were compared with the dose distribution measured using a film as ground truth. The comparison was quantitatively conducted using a gamma evaluation method [18] with three criteria of dose difference/distance-to-agreement: $3 \% / 3 \mathrm{~mm}, 2 \% / 2 \mathrm{~mm}$, and $1 \% / 1 \mathrm{~mm}$. The minimum dose threshold was chosen as $10 \%$.

\section{Results}

We obtained two dose distributions calculated using kVCT and hybrid CT, and one dose distribution measured using a film per patient. Fig. 5 shows the three-dose distributions in patient 1. Comparing Fig. 5a and 5b, different dose distribution patterns (see black arrows) are observed. The pattern in hybrid CT is closer to the measured pattern than that observed in $\mathrm{kV}-\mathrm{CT}$.

Table 1 presents the evaluated gamma pass rates between the calculated and measured dose distributions, and the plots are shown in Fig. 6. The gamma pass rates decreased as the gamma criteria were strengthened in all patients. The pass rate dropped significantly at the $1 \% / 1 \mathrm{~mm}$ criterion. The pass rate of hybrid CT was higher than that of $\mathrm{kV}-\mathrm{CT}$ in all cases, which remained above $80 \%$ even at the criterion of $1 \% / 1 \mathrm{~mm}$. 
Table 1. Gamma pass rates between the calculated and measured dose

\begin{tabular}{|c|c|c|c|c|c|c|c|c|c|}
\hline \multirow{2}{*}{ Gamma criteria } & \multicolumn{2}{|c|}{ Patient 1} & \multicolumn{2}{|c|}{ Patient 2} & \multicolumn{2}{|c|}{ Patient 3} & \multicolumn{3}{|c|}{ Mean } \\
\hline & $\mathrm{kV}-\mathrm{CT}$ & Hybrid CT & kV-CT & Hybrid CT & $\mathrm{kV}-\mathrm{CT}$ & Hybrid CT & $\mathrm{kV}-\mathrm{CT}$ & Hybrid CT & $\Delta$ \\
\hline $3 \% / 3 \mathrm{~mm}$ & 99.1 & 99.9 & 100.0 & 100.0 & 97.8 & 99.8 & 99.0 & 99.9 & 0.9 \\
\hline $2 \% / 2 \mathrm{~mm}$ & 97.3 & 99.6 & 97.2 & 99.2 & 91.7 & 98.3 & 95.4 & 99.0 & 3.6 \\
\hline $1 \% / 1 \mathrm{~mm}$ & 74.3 & 91.6 & 75.8 & 85.0 & 70.9 & 84.4 & 73.7 & 87.0 & 13.3 \\
\hline
\end{tabular}

Values are presented as percentage only.

$\mathrm{kV}$-CT, kilovoltage computed tomography; $\Delta$, difference in the average gamma pass rates between hybrid CT and $\mathrm{kV}$-CT (in percentpoint).
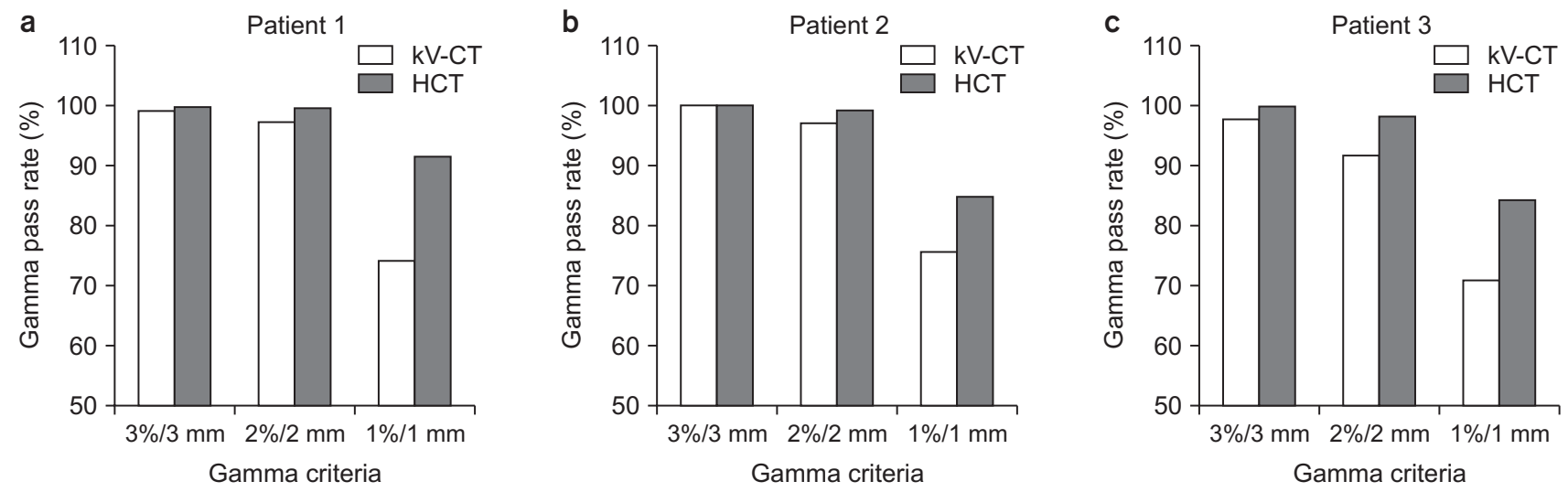

Fig. 6. Comparison of gamma pass rates at different gamma criteria in the three patients. kV-CT, kilovoltage computed tomography; HCT, hybrid CT.

\section{Discussion}

Although the $3 \% / 3 \mathrm{~mm}$ gamma criterion has been widely used for the verification of RTP, it is recommended to use stricter criteria because of the development of RTP techniques that require high accuracy and precision, such as intensity-modulated radiation treatment or stereotactic body radiation treatment $[19,20]$. In this study, all pass rates obtained using the $3 \% / 3 \mathrm{~mm}$ gamma criterion, which was 99.0\% (kV-CT) and 99.9\% (Hybrid CT) on average, were sufficient to be accepted for treatment. When the $2 \% / 2 \mathrm{~mm}$ criterion was used, the hybrid CT showed significant superiority over kV-CT (Fig. 6). When the $1 \% / 1 \mathrm{~mm}$ criterion was used, the difference in gamma pass rates between them was about 13\%p. These results indicate that hybrid CT, which can reduce artifacts due to metal prostheses, can aid accurate dose calculation for RTP. For instance, severe metal artifacts are frequently observed in head and neck CT images owing to metallic dental prostheses. Because the important organs at risk, such as the brain stem, optic nerves, and parotid glands, are located close to each other in the head and neck, an accurate and precise RTP is necessary. Thus, hybrid CT plays a significant role in making RTP for the head and neck.

Fig. 7 shows the gamma evaluation results of patient 1 . Comparing Fig. 4 and 7, using kV-CT, we observe that the distribution of gamma failures where gamma values are greater than 1 approximately coincides with the distribution of metal artifacts (see black dotted boxes in Fig. $4 \mathrm{a}$ and 7). However, using hybrid CT, it can be visually verified that both image artifacts and gamma failures are significantly reduced.

Established MAR techniques that could help only in the reduction of image artifacts but not in image restoration, like hybrid CT, were not considered since the restored images were more helpful for target/organ identification and contouring. In our previous study [14], the hybrid CT was compared with three well-known MAR techniques, such as linear interpolation, Lagrange interpolation with high-order polynomials, and normalization prior to interpolation (NI) with the best performance among them. We compared the hybrid CT performance to NI. The difference in their dose 


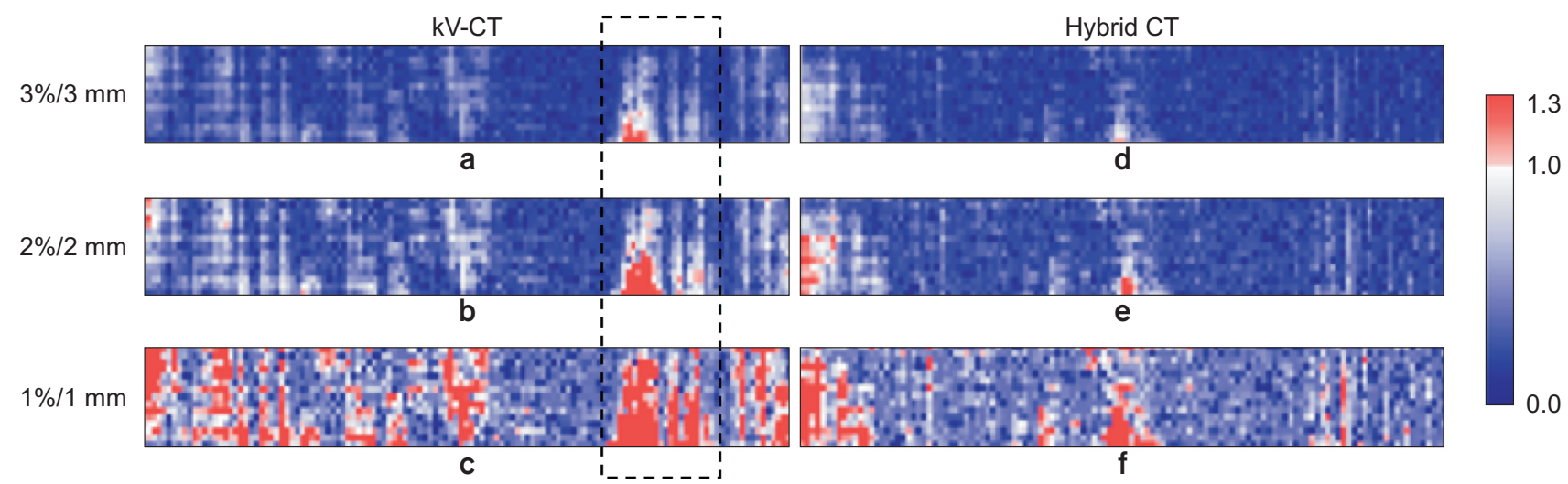

Fig. 7. Gamma evaluation results of (a-c) kilovoltage computed tomography (kV-CT) and (d-f) hybrid CT with the three gamma criteria in patient 1.

calculation seems to be worthy for further study to evaluate the effects of secondary artifacts that may occur during the hybrid CT generation.

In this study, the hybrid CT performance was first verified for the head and neck. The same verification will be implemented for other sites where metallic prostheses can be used, such as artificial hip joints or surgical clips. Besides, verification using a phantom that more closely mimics human anatomy instead of using a cylindrical phantom may be necessary.

\section{Conclusions}

RTP should be carefully constructed when metal artifacts are present in $\mathrm{kV}$-CT images considering their non-negligible effect on dose calculation. We expect that using hybrid CT can be a suitable approach to avoid the effect of severe metal artifacts on dose calculation and contouring.

\section{Acknowledgements}

This research was supported by the Basic Science Research Program through the National Research Foundation of Korea (NRF) funded by the Korean government (2017R1D1A1B03031351), and by a 2020 research grant from Pusan National University Yangsan Hospital.

\section{Conflicts of Interest}

The authors have nothing to disclose.

\section{Availability of Data and Materials}

All relevant data are within the paper.

\section{Author Contributions}

Conceptualization: Hosang Jeon and Jiho Nam. Data curation: Dong Woon Kim and Dahl Park. Formal analysis: Dong Woon Kim and Dahl Park. Funding acquisition: Yongkan Ki and Jiho Nam. Investigation: Ji Hyeon Joo and Yongkan Ki. Methodology: Hosang Jeon and Dong Woon Kim. Project administration: Hosang Jeon. Resources: Yongkan Ki and Wontaek Kim. Software: Yongkan Ki and Dong Hyeon Kim. Supervision: Wontaek Kim and Yongkan Ki. Validation: Hosang Jeon and Dong Hyeon Kim. Visualization: Ji Hyeon Joo and Dong Hyeon Kim. Writing-original draft: Hosang Jeon. Writing-review \& editing: Hosang Jeon and Yongkan Ki.

\section{References}

1. Glover GH, Pelc NJ. An algorithm for the reduction of metal clip artifacts in CT reconstructions. Med Phys. 1981;8:799807.

2. Yazdi M, Gingras L, Beaulieu L. An adaptive approach to metal artifact reduction in helical computed tomography for radiation therapy treatment planning: experimental and clinical studies. Int J Radiat Oncol Biol Phys. 2005;62: 1224-1231.

3. Zhao S, Robertson DD, Wang G, Whiting B, Bae KT. X-ray 
CT metal artifact reduction using wavelets: an application for imaging total hip prostheses. IEEE Trans Med Imaging. 2000;19:1238-1247.

4. Kalender WA, Hebel R, Ebersberger J. Reduction of CT artifacts caused by metallic implants. Radiology. 1987;164:576577.

5. Mahnken AH, Raupach R, Wildberger JE, Jung B, Heussen N, Flohr TG, et al. A new algorithm for metal artifact reduction in computed tomography: in vitro and in vivo evaluation after total hip replacement. Invest Radiol. 2003;38:769775.

6. Yu H, Zeng K, Bharkhada DK, Wang G, Madsen MT, Saba $\mathrm{O}$, et al. A segmentation-based method for metal artifact reduction. Acad Radiol. 2007;14:495-504.

7. Zhang Y, Zhang L, Zhu XR, Lee AK, Chambers M, Dong L. Reducing metal artifacts in cone-beam CT images by preprocessing projection data. Int J Radiat Oncol Biol Phys. 2007;67:924-932.

8. Boas FE, Fleischmann D. Evaluation of two iterative techniques for reducing metal artifacts in computed tomography. Radiology. 2011;259:894-902.

9. Mehranian A, Ay MR, Rahmim A, Zaidi H. X-ray CT metal artifact reduction using wavelet domain L0 sparse regularization. IEEE Trans Med Imaging. 2013;32:1707-1722.

10. Zhang X, Wang J, Xing L. Metal artifact reduction in X-ray computed tomography (CT) by constrained optimization. Med Phys. 2011;38:701-711.

11. Stayman JW, Otake Y, Prince JL, Khanna AJ, Siewerdsen JH. Model-based tomographic reconstruction of objects containing known components. IEEE Trans Med Imaging. 2012;31:1837-1848.

12. Ruchala KJ, Olivera GH, Schloesser EA, Mackie TR. Mega- voltage CT on a tomotherapy system. Phys Med Biol. 1999; 44:2597-2621.

13. Meeks SL, Harmon JF Jr, Langen KM, Willoughby TR, Wagner TH, Kupelian PA. Performance characterization of megavoltage computed tomography imaging on a helical tomotherapy unit. Med Phys. 2005;32:2673-2681.

14. Jeon H, Park D, Youn H, Nam J, Lee J, Kim W, et al. Generation of hybrid sinograms for the recovery of kV-CT images with metal artifacts for helical tomotherapy. Med Phys. 2015;42:4654-4667.

15. Ahnesjö A, Aspradakis MM. Dose calculations for external photon beams in radiotherapy. Phys Med Biol. 1999;44:R99R155.

16. Ahnesjö A. Collapsed cone convolution of radiant energy for photon dose calculation in heterogeneous media. Med Phys. 1989;16:577-592.

17. Lu W, Olivera GH, Chen ML, Reckwerdt PJ, Mackie TR. Accurate convolution/superposition for multi-resolution dose calculation using cumulative tabulated kernels. Phys Med Biol. 2005;50:655-680.

18. Low DA, Harms WB, Mutic S, Purdy JA. A technique for the quantitative evaluation of dose distributions. Med Phys. 1998;25:656-661.

19. Heilemann G, Poppe B, Laub W. On the sensitivity of common gamma-index evaluation methods to MLC misalignments in Rapidarc quality assurance. Med Phys. 2013;40: 031702.

20. Fredh A, Scherman JB, Fog LS, Munck af Rosenschöld P. Patient QA systems for rotational radiation therapy: a comparative experimental study with intentional errors. Med Phys. 2013;40:031716. 